\title{
Design of Intercom System in Stage Video Surveillance and Scheduling System \\ Yihua $\mathrm{Li}^{1, \mathrm{a}}$, Xiaodong Zhang ${ }^{1, \mathrm{~b}}$, Zhicong Feng ${ }^{2, \mathrm{c}}$, ,Zhenhui Luan $^{1, \mathrm{~d}}$ \\ ${ }^{1}$ School of Mechanical Engineering, Anhui University of Science and Technology, Huainan Anhui, 232001, China \\ ${ }^{2}$ School of Electric and Information Engineering, Anhui University of Science and Technology, Huainan Anhui, 232001, China

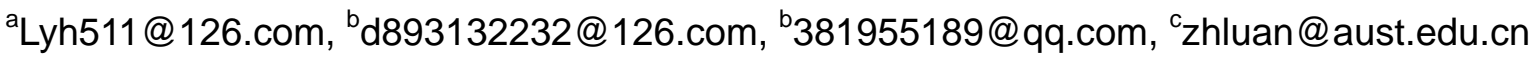

Keywords: stage; schedule; intercom system; VolP

\begin{abstract}
Through the old intercom system for stage could fulfill all types of work in performances, but could not escape the limitations of traditional techniques for the reason the core of this whole system is still based on circuit-switched technology. Networked intercom system for stage dispatch is proposed for overcoming the shortcomings of old intercom system. It can be achieved by using VoIP technology through the SIP control signaling protocol in stage video surveillance and scheduling system. The composition and technical characteristics of various parts in system are analyzed, and the way to achieve networking intercom system is introduced. Our study have shown that the various parts of system connect more closely and there is a very important practical significance in solving the deficiencies of the current intercom system for stage, after networked intercom system achieves which is based on stage video surveillance and scheduling system.
\end{abstract}

\section{Introduction}

Theater intercom system, mainly used for the background personnel's communication, is the most complicated and important subsystem in stage video surveillance and scheduling system. Party-line technology is given priority to, and the development of the circuit switching technology has stalled. So various business is confused, causes each equipment is made by different manufacturers. In traditional stage video surveillance and scheduling system, the intercom system and the video monitor system often exist as a separate system. The cable and the wireless intercom system work with different hosts. So the system becomes complex. Operation become cumbersome and easily go wrong. System maintenance is very inconvenient. In general, most hosts of system use simplified single intercom system. It cannot communicate point to point, so a stage manager often speaks to many people. So target is ambiguous and not enough outstanding. And cable communications are vulnerable for complex circuit and distance, wireless communications are susceptible for interference and signal source, blind area. Therefore, the research of networked intercom system integration and the seamless integration of all parts, it is necessary for solving the defects of theater intercom communication and scheduling.

\section{The Design of the Intercom System}

Theater intercom system mainly includes three parts: the cable system, wireless system and public broadcast. they are closely connected together through internal LAN. 


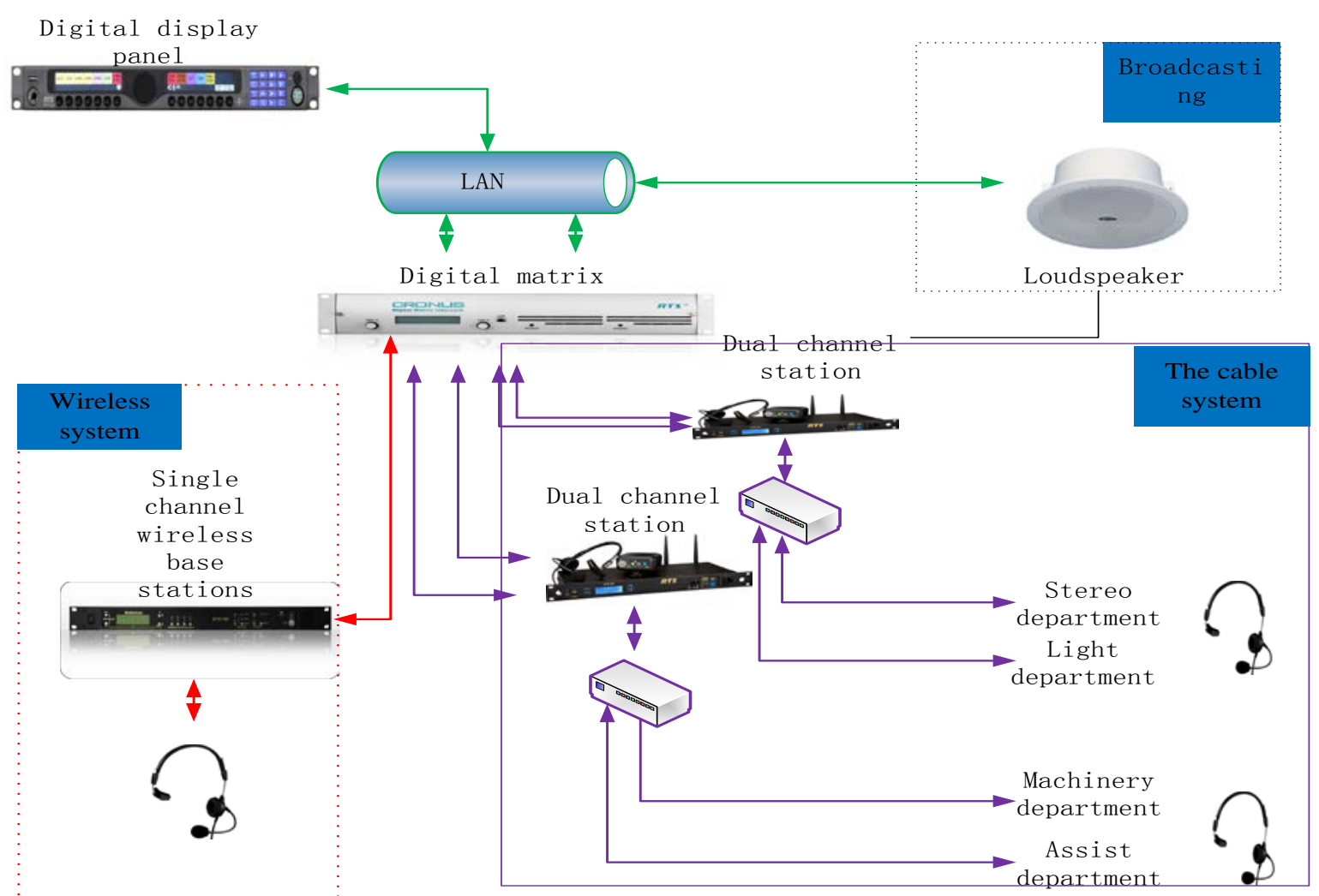

Fig.1, The intercom system diagram.

The diagram of intercom system is shown in figure 1. The system is divided three parts: cable intercom, wireless intercom and public broadcast. Terminal through IP network connection to the end of each part on the switch, the exchange of information in the form of IP packet. System of the equipment monitoring and scheduling system exists in the stage of subnet, in order to distinguish with video surveillance equipment and network, convenient scheduling system builds and stage supervision.

\section{Important parts of the intercom system}

Voice signal is sampled, quantized and coded by digital matrix. Each digital signal is deal with TDM time-division multiplexing, then after TDM releasing multiplexing and time-slot interchange. Each digital signal is routing to specify listen channels[1]. So information source become information sink. 32 channel distribution could be accomplished by the digital matrix, CRONUS could work with VoIP card. And it has IP connectivity and network remote monitoring Settings; It has a very convenient data connection in front panel. Its advantages are high quality, strong software functions, small size, flexible configuration. The functional block diagram of digital matrix is shown in figure 2 .
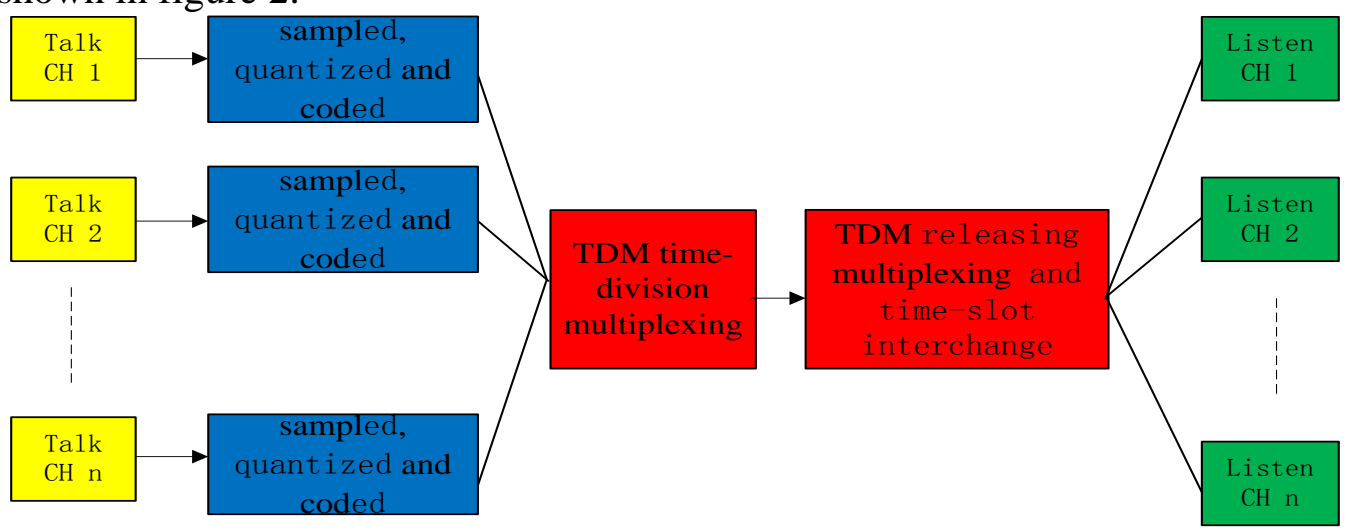

Fig.2, The functional block diagram of digital matrix.

Features: visual graphical interface, convenient operation in front panel. It has an visual user interface and colors function panel. It is designed in conforming to Human body engineering and 
easily to install in any room. It has three auxiliary inputs. They can be used in independent control function and audio routing,and can realize more functions through adding palette. It uses DSP in eliminating echo, and sound equalization, sound mixing, sound filtering, and sound measurement.

\section{The cable intercom.}

It has reliable full-duplex communication and could work with eight full- duplex mode communication pockets. When many people work at same time, It still could play a good role. In the system, TR - 240 pocket could work in cable mode, and it is very convenient in this mode. Features:it has multi-level security and audio data encryption,and it could select automatically clear, less disturbance channel. And we could read real-time working condition about TR-240 pocket from liquid crystal display.

\section{Wireless system}

Wireless system born on the basis of wireless system,and gradually develop and become perfect. The biggest advantage of this system is that users could communicate freely in a certain area. It can work in connecting digital Matrix in system. The system contains a base station and wireless pockets, they are adjustable in frequency. Features: users could watch clearly working status of the pockets from back-lit LCD in the base station. Each base station could hold four duplex pockets, and can be used as a "personal" to talk and listen. It could meet the conversation requirements in work process[2].

\section{Network of Internal System}

The server sets two keys in advance. The call key is used to call manager, the group key is used in talking in group. There are Ethernet ports where we can access, it is greatly convenient to timely communicate.. Wireless system sets up a wireless router, mobile terminals could talk in it with wifi transmission technology, cooperating with wireless base station and walkie-talkie. According to internal structures characteristics of theater, wireless signal is covered reasonably. Some mobility departments, such as art group, its members could talk with mobile terminals in wireless signal coverage area. Broadcasting terminals and active speakers connect system through core switch, stage managers could speak in internet and selectively order instructions to different partitions[3].

\section{Network of Control Center}

Control center is the core part of internal system. It is divided into modules: call service module, media control module and management module. Call service module is control system of the whole system and can control unified call. It includes call control program and call analysis program. Call control program receives call control information from protocol adaptation layer, so it could establish the call. Then call analysis program works for routing and addressing. When users call terminal, it could according to the corresponding database data to establish the corresponding IP address. And this IP address is added to IP packets and the best route will be selected to reduce transmission delay. Control module is mainly used to encapsulate real-time media data. Data is input in constant speed, in order to make multimedia stream with a uniform, continuous flow form to reach, as far as possibly improve performance. Management module can realize user registration and dynamic grouping management, permissions and safety management. At the same time it also monitor running state of other sub-modules and evaluate system operation resources[4].

\section{Technology of VoIP}

VoIP (Voice Over IP) codes and compresses voice signal and then converts it into IP packet which is transported in internet. The call need streaming media technology to ensure media continuous, real-time transmission and proper signaling technology to ensure[5]. SIP is selected as signaling protocol in system, it can be used to create, change and terminate conversation between users based on IP network[6]. It could work with unicast and multicast. It has two elements : SIP user agent and SIP network server. User Agent is responsible for sending a request to server, which is responsible for responding to requests issued by the agent. A call starts from the user agent and terminates at the server.

\section{Summary}

Network integration of intercom system is built on the basis of network of control center and 
network of intercom system. It realizes wireless systems and cable system integrated, cable and wireless hybrid intercom, point to point and point to multipoint communication. It is easier to operate and maintain, and the complexity of system and the difficulty of construction is also declined. The audio and video technology, multimedia technology and computer network technology are used to build an IP network integrated system, so that the various parts of system connect more closely. It is convenient to communicate and enhance the effect of performance.

\section{Acknowledgments}

This work is supported by National Science and Technology Support Key Project. Present key support technology research and development and to demonstrate the application performance(No.2012BAH38F00).

\section{References}

[1] Weidong Gu. MODERN TV TECHNOLOGY. NO.2(2005). P. 53 56(In Chinese).

[2] Haiyan He. INFORMATION TECHNOLOGY. NO.2(2012). P.172 173(In Chinese).

[3] Hao Wang. ART SCIENCE AND TECHNOLOGY. NO.2(2008). P.22 24(In Chinese).

[4] Jianlong Cao. Design and implement of mobile VoIP HD intercom system based on android[D]. Nanjing: Nanjing University of Science and Technology, 2013: 21 50(In Chinese).

[5] Lijie Fang, Yu Liu.SCIENCE MOSAIC. NO.3(2010). P.42 45(In Chinese).

[ 6 ] Yinxing Chen, Liqing Zhou. MODERN SCIENCE \& TECHNOLOGY OF TELECOMMUNICATIONS. NO.10(2006). P.11 15(In Chinese). 